\title{
Late distant metastasis from papillary carcinoma of thyroid gland
}

\begin{abstract}
Objective: How long we have to follow the case of papillary carcinoma of thyroid gland. The present case report will reveal the late appearance of distant metastasis in a case of papillary carcinoma of thyroid gland. This was confirmed by frequent thyroglobulin(tg) assessment for a case of papillary carcinoma in the thyroid gland of a young lady, after fourteen years. When we noticed a substantial elevation of tg., a PET scan revealed a focus of abnormal uptake in right side of her neck. We performed a modified radical right neck dissection, preserving the sternocleidomastoid muscle and jugular vein and spinal accessory nerve as well. The permanent histopathological studies showed metastatic papillary carcinoma within five lymph nodes
\end{abstract}

Keywords: papillary carcinoma, PET scan, metastasis
Volume 10 Issue I - 2019

\author{
Kamran Aryana,' Rasoul Zakavi,' N Mokhtari \\ Amir Majdi \\ 'Nuclear Medicine Research Center, Ghaem Hospital, Mashhad \\ University of Medical Sciences, Iran \\ 2ENT Department, Ghaem Hospital, Mashhad University of \\ Medical Sciences, Iran
}

Correspondence: N Mokhtari Amir Majdi, Prof. of Otolaryngology/ Head and Neck Surgery, Ghaem Hospital, Mashhad University of Medical Sciences, Iran, Emailmokhtarinematollah@yahoo.com

Received: November 04, 2017 | Published: February 14, 2019

\section{Report of case}

A forty two years old lady, underwent a total thyroidectomy, fourteen years ago for a mass in the thyroid gland with positive FNA for papillary carcinoma. She did not have enlarged lymph node and had an uneventful post operative course. Her histopathological report was also positive for papillary carcinoma. Due to the size of tumour, she had a course of radioactive iodine (1100 MBq in september 2002). From then on she was on annual follow up and had undetectable serum tg and negative Anti-tg AB. She was on supressive therapy with thyroid hormones with $\mathrm{TSH}$ level $<0.5 \mathrm{mU} / \mathrm{L}$. Annual neck ultrasonography was negative for any suspicious cervical lymph nodes. She had uneventful pregnancy and had breast feeding for two years. Two years ago she underwent whole body iodine scan which was negative for any iodine avid focus, anyhow stimulated tg level was $30 \mathrm{ng} / \mathrm{ml}$. Ultrasonography was negative and she did not accept further studies

During her last three tg assessment, we noticed a substantial raise in $\mathrm{tg}$. So we decided to ask for a whole body 18FDG-PET/ST. This revealed a hypermetabolic activity in her right mid cervical lymph node (SUV max=36). She was taken to the operating room and a modified neck dissection was performed, saving her sternocleidomastoid muscle and jugular vein as well as her spinal accessory nerve. Five of the lymph nodes were positive for papillary thyroid carcinoma. She will be followed again on her previous schedule.

Our protocol to follow the case of papillary carcinoma of the thyroid gland has been to perform a whole body scan imaging 3-14 days after treatment, and check that TSH suppresion is below $0.1 \mathrm{mu} / 1$ for high and intermediate risk patients, and $0.1-0.5 \mathrm{mu} / 1$ for Low risk patients. We make periodic determination (every 6-12months) of serum thyroglobuline in hypo-Thyroid state or after thTSH.

At twelve month, we perform a diagnostic whole body iodine scan after hormone withdrawal or thTHS. This is appropriate for high and intermediate risk patients. The interval between treatment for patients with metastatic thyroid carcinoma depends on the response to the previous treatment and also on the extent and Location of the metastatic disease.

\section{Discussion}

Late metastatic presentation of papillary carcinoma is well known. ${ }^{1,2}$ The latest one was reported by Tucker. ${ }^{2}$ The anatomic region of metastasis can be anywhere, lungs, bones, ${ }^{1}$ optic nerve ${ }^{3}$ and lymph nodes of neck, as in our case we are presenting. The long term follow up was also recommended by frequent $t g$ studies. This was performed by us and was the only possible way to early diagnosis of distant metastasis, which was confirmed using PET scan studies.

Our method of management (total thyroidectomy and I-131 ablation) is recommended by different authorities. ${ }^{1-4}$ The annual assessment of $\mathrm{tg}$ is also recommended by different authorities for early detection of obscure distant metastasis. Our case, while being symptom free was detected by $\mathrm{tg}$ assessment. When we were faced with three subsequent elevation of $\mathrm{tg}$, we decided on a whole body FDG PET/CT scanning which confirmed the diagnosis and the position of distant metastasis in neck. The whole body scanning can be performed by I- 131 and in case of negative findings by 18FDG $\mathrm{PET} / \mathrm{SC}$.

This method of study is also useful to rule out multifocal metastatic lesions. In such cases, surgery was not recommended and further treatment depends on I- 131 ablation. A review of different articles to revisit our method of treatment and follow up ${ }^{4}$ confirmed that this case was methodically managed. Gharib and Hurely also recommended this approach for treatment and follow up of patients with ordinary papillary carcinoma of the thyroid gland. Although patients who had anaplouidy in their chromatin, behave differently, this study was not performed for our case.

Confronting cases like this, raises the question" how long we have to continue our follow up for these patients?." We believe to continue this method of management throughout their life. 


\section{Acknowledgments}

None.

\section{Conflicts of interest}

None.

\section{References}

1. Mathew D. Ringle. Thyroid. 2011;487-492.
2. Miriam E. Thyroid carcinoma can recur and kill decades later, presented before Endocrin surgeons. 2013.

3. M Amir Ahmadi, Denos Nicholes, Bita Esmaeli. Late choroidal metastasis secondary to papillary thyroid carcinoma. American journal of ophthalmology. 2014.

4. Daniel L Hurely, Hossein Gharib. The otolaryngic clinics of North America. 1996 